\title{
The 'early pregnancy factor' revisited: the effect of ammonium sulfate on the capacity of pregnant mouse sera to express activity in the rosette inhibition assay
}

\author{
I. Q. Clark, C. Orozco, I. E. Cock and F. M. Clarke* \\ Faculty of Science and Technology, Griffith University, Nathan, Brisbane, 4111, Australia
}

\begin{abstract}
The effect of ammonium sulfate on the capacity of sera of pregnant animals to induce the expression of increased rosette inhibition titres in the rosette inhibition assay, that is, to express the so-called early pregnancy factor activity, was reinvestigated. The results show that the sera of pregnant mice contain low molecular mass (less than I $\mathrm{kDa}$ ) moieties active in the rosette inhibition assay. Some of these moieties could be removed from the macromolecular components of sera by dialysis; however, most, or at least the most potent, of these molecules were shown to be associated with macromolecular components of the sera and were not removed by dialysis. Treatment of sera of pregnant mice with $40 \%$ ammonium sulfate released the bound low molecular mass moieties and these moieties partitioned into the supernatant fraction, whereas the macromolecular components to which they were bound partitioned into the pellet fraction. Extensive dialysis removed the low molecular mass active moieties from the supernatant fraction. The macromolecular components remaining in the supernatant retentate fraction obtained after extensive dialysis counteracted the action of the low molecular mass moieties in a dose-dependent manner in inducing increased rosette inhibition titres. However, macromolecular components in the extensively dialysed pellet fraction associated with the low molecular mass moieties in the absence of ammonium sulfate and modified their dose-response characteristics in the biological assay. The macromolecular components in the extensively dialysed pellet and supernatant retentate fractions alone could not increase rosette inhibition titres; however, when they were recombined, components in the extensively dialysed pellet retentate fraction cooperated with components in the extensively dialysed supernatant retentate fraction to allow for the expression of activity. These results are considered in the context of a new model of the system of components present in sera of pregnant mice which allow for the expression of so-called early pregnancy factor activity.
\end{abstract}

\section{Introduction}

The ability of early pregnancy sera to act on lymphocytes to cause increased rosette inhibition titres in the rosette inhibition assay has been studied for a considerable time (Smart et al., 1981; Whyte and Heap, 1983; Morton, 1984; Chard and Grudzinkas, 1987; Morton et al., 1987). This activity of pregnancy sera can be detected within a few hours of fertilization, making it the earliest known systemic sign that fertilization has taken place (Morton et al., 1974, 1976, 1977; Rolfe, 1982; Bessho et al., 1984; Mesrogli et al., 1988). Its discovery, in the mid-1970s, was probably the first indication that there were significant communications between mother and embryo before implantation. However, even now a complete molecular definition of this activity is not available. Shortly after its discovery, this activity was ascribed (Morton et al., 1977) to the presence of an 'early pregnancy factor' in serum

*Correspondence.

Received 24 May 1993. of pregnant animals, and this factor was assumed to be a pregnancy-specific protein (Rolfe, 1982; Morton, 1984; Morton et al., 1987; Mehta et al., 1989). However, even the early studies indicated that any such protein must be complex (Clarke et al., 1978, 1980; Morton et al., 1980; Rolfe et al., 1983), and Clarke and Wilson (1985) suggested that a complex set of components might interact to produce this biological activity. Attempts to isolate a protein responsible for early pregnancy factor activity have produced a variety of results without any convincing identification or characterization (Wilson et al., 1983; Cavanagh, 1984, 1985; Bose et al., 1989; Mehta et al., 1989; Sueoka et al., 1989a; Klima et al., 1992).

Studies on the nature of some of the molecules and mechanisms involved in the rosette inhibition assay (Orozco et al., 1986, 1990; Clarke et al., 1990a, b) that allow the expression of increased rosette inhibition titres and also on some of the molecules and mechanisms by which pregnancy sera achieve this effect (Clarke et al., 1987, 1991; Di Trapani et al., 1991) have provided some new insights. Characterization of human 
(Di Trapani t al., 1991) and ovine (Clarke et al., 1991) placental preparations, active in the rosette inhibition assay, revealed the presence of a polypeptide of relative molecular mass $12 \mathrm{kDa}$ with associated active moieties of low molecular mass. Aminoterminal sequence analyses indicated that the proteins of $M_{\text {r }} 12000$ were vertebrate thioredoxins, an identification confirmed by cloning and sequencing of the cDNA. Human recombinant thioredoxin has been expressed in Escherichia coli and purified. Pure thioredoxin alone does not induce increased rosette inhibition titres. However, when it is applied to spleen cells in combination with, or subsequent to such cell stimuli as platelet-activating factor (PAF) or normal serum, thioredoxin plays a permissive role allowing for the expression of increased inhibition titres, where none are achieved in its absence. It has been shown to achieve this effect by preventing, or reversing, a refractory state induced in the spleen cell population by these stimuli, allowing lipoxygenase-dependent products, also generated in response to these stimuli, or possibly naturally present in the case of sera, to exert their effects in inducing increased rosette inhibition titres (Clarke et al., 1991).

Clarke et al. (1991) showed that all sera could stimulate the spleen cells used in the assay to produce potentially active moieties. However, as only sera of pregnant animals result in increased rosette inhibition titres, it has been suggested that sera from pregnant animals may be distinguished from that from non-pregnant animals by the presence of functional forms of thioredoxin or thioredoxin-like molecules, which act to reverse or prevent the refractory state induced by other serum components, and so allow for increased inhibition titres. Adsorption studies with anti-thioredoxin antibody (Clarke et al., 1991) support these conclusions and also indicate that there probably is an association in pregnancy sera (but not nonpregnancy sera) between the thioredoxin-like proteins and some active moieties of low molecular mass. Thus, it seems that the capacity to induce increased rosette inhibition titres is not due to the presence in sera of pregnant animals of some unique, pregnancy-specific protein but rather that all sera contain, or at least possess the capacity to generate, mixtures of active and inhibitory moieties, that may induce increased inhibition titres depending on the relative proportions of these antagonistic moieties and the presence or absence of specific functional forms of thioredoxin molecules that, if present, allow the expression of increased rosette inhibition titres.

Although this new view of the ability of pregnancy sera to induce increased rosette inhibition titres is supported by many recent observations, reviewed by Clarke (1992), it is necessary that it also explains some of the unusual properties previously ascribed to early pregnancy factor. Paramount amongst these are the apparent association of this activity with species of multiple molecular mass in sera of pregnant animals (Clarke et al., 1978, 1980; Rolfe et al., 1983) and the susceptibility of this activity to ammonium sulfate fractionation of sera (Clarke et al., 1980; Sueoka et al., 1989b). While the first mentioned phenomenon is addressed in a later paper, the present report re-examines the latter phenomenon. Clarke et al. (1980) reported that when pregnancy sera were treated with $40 \%$ ammonium sulfate, and the resulting supernatant and pellet fractions were collected and extensively dialysed and then tested in the rosette inhibition assay, neither pellet, nor supernatant fraction, could induce increased rosette inhibition titres. However, when these dialysed fractions were recombined the combination induced increased inhibition titres, mimicking the action of the unfractionated sera. At the time, these data were taken to suggest that there were two parts to the system that allows pregnancy serum to induce increased inhibition titres. The supernatant fraction was said to contain the $\mathrm{A}$-component(s) and the pellet fraction the B-component(s). Contemporaneous studies (Morton et al., 1980) on the tissues involved in production of early pregnancy factor activity suggested that the ovaries of pregnant animals produced components, functionally equivalent to those in the pellet fraction from pregnancy sera, while the oviducts produced component(s) functionally equivalent to those in the supernatant fraction. As a result of these and other studies, it was suggested (Morton, 1984; Morton et al., 1987) that the proposed early pregnancy factor molecule was made up of two components or subunits. While discussion of this two component system continues (but see recent modified view of Cavanagh et al., 1991), little definitive evidence to support this simple concept of early pregnancy factor has been forthcoming. If the model of the early pregnancy factor system recently proposed (Clarke et al., 1991; Clarke, 1992) is correct, it should provide an alternative and substantiated explanation of this phenomenon. The work described here reinvestigates the effect of ammonium sulfate fractionation on the capacity of sera from pregnant animals to induce increased rosette inhibition titres to determine whether the observations are consistent with this new model. While this report was in preparation, a paper by Cavanagh et al. (1991) appeared in which this effect of ammonium sulfate was also reassessed. The present report takes account of the new observations of Cavanagh et al. (1991), and in so doing a number of issues that arise from a comparison of their observations and interpretations and those presented in this report are addressed.

\section{Materials and Methods}

\section{Collection of sera}

Blood was collected from male and female mice and the serum separated as described by Orozco ef al. (1986). All animals were mature Quackenbush mice, 6-12 weeks of age. Stages of the oestrous cycle and stage of pregnancy were identified as described by Orozco et al. (1986).

\section{Ammonium sulfate fractionation}

Individual serum samples were brought to $40 \%$ saturated ammonium sulfate, either by adding solid ammonium sulfate (at a rate of $0.242 \mathrm{~g} \mathrm{ml}^{-1}$ of sample) or by adding an equal volume of a $2 \times 40 \%$ saturated ammonium sulfate solution (prepared by dissolving ammonium sulfate at a rate $0.484 \mathrm{~g}$ $\mathrm{ml}^{-1}$ of water). After standing at $4^{\circ} \mathrm{C}$ for 30 minutes, the samples were centrifuged at $23000 \mathrm{~g}$ for $5 \mathrm{~min}$. The supernatant fractions were collected and the pellet fractions were redissolved in a volume of PBS, equivalent to the original sample volume. Aliquots $(5 \mu \mathrm{l})$ were stored for later analyses, and the supernatants and redissolved pellet fractions were dialysed as described below. 


\section{Dialysis}

Dialysis was performed using either nominal $8 \mathrm{~K}$ (Union Carbide Co., Chicago, IL) or $1 \mathrm{kDa}$ cut-off (Spectrapor) tubing. In all experiments, except that designated as dialysis Expt 2, individual samples were dialysed in separate containers (beakers) against 100-fold excess volumes of PBS (for example, $0.5 \mathrm{ml}$ samples were dialysed against $50 \mathrm{ml}$ of PBS). Three successive dialysis steps were used, the first for $4 \mathrm{~h}$ at room temperature $\left(20-23^{\circ} \mathrm{C}\right)$, the second against a fresh batch of PBS at $4^{\circ} \mathrm{C}$ overnight $(16 \mathrm{~h})$, and the third, again with fresh PBS, at room temperature for $4 \mathrm{~h}$. After each step, aliquots of the retentates and dialysates were stored for later analysis. In dialysis Expt 2, the supernatant and pellet fraction derived from a given serum were dialysed together in the same container. Specific details of the individual dialysis experiments are described in the relevant results section.

\section{Spleen cell preparations, cell incubations with sample and the rosette inhibition assay}

Mouse spleen cell preparations were prepared as described by Orozco et al. (1986). For the standard cell incubation with sample before testing in the rosette inhibition assay, $0.3 \mathrm{ml}$ aliquots of cell suspension $\left(4.5 \times 10^{6}\right.$ cells) were centrifuged at $300 \mathrm{~g}$ for $5 \mathrm{~min}$. The cell pellets were resuspended in PBS to give a final volume of $80 \mu \mathrm{l}$ after addition of test samples. Sera or fractions derived from them were added as $2 \mu \mathrm{l}$ aliquots; these samples were serially diluted in PBS-BSA $\left(0.2 \mathrm{mg} \mathrm{ml}^{-1}\right)$ as required. After incubation at $37^{\circ} \mathrm{C}$ for $30 \mathrm{~min}$, the cells were sedimented and then washed twice in $1 \mathrm{ml}$ Hank's balanced salt solution (HBSS; Gibco, Grand Island, NY). After the final centrifugation, at $300 \mathrm{~g}$ for $5 \mathrm{~min}$, the cells were resuspended in a final volume of $0.5 \mathrm{ml}$ and dispensed into the antilymphocyte serum dilutions. The rosette inhibition assay to determine the rosette inhibition titre was performed as described by Orozco et al. (1986) and Clarke et al. (1987), except that all reagent volumes (anti-lymphocyte serum, cells, complement, HBSS, and red blood cell suspension) were halved. The rosette inhibition titre was expressed as $\log _{2}$ (reciprocal dilution of anti-lymphocyte serum $\times 10^{-3}$ ) (Rolfe et al., 1984). Thus, if rosette formation is inhibited to an anti-lymphocyte serum dilution of 1 in $4 \times 10^{6}$, the inhibition titre is expressed as 12 . This inhibition titre of 12 was consistently obtained on spleen cells incubated in PBS alone; consequently, the observation of rosette inhibition titre greater than 16 was considered to be significantly higher than the control, and represents an increased inhibition titre.

\section{Results and Discussion}

\section{Release of low molecular mass active moieties by ammonium sulfate fractionation of sera of pregnant animals}

In agreement with the observations of Clarke $e t$ al. (1980), it was found that when sera of pregnant mice were fractionated with $40 \%$ ammonium sulfate to yield supernatant and pellet fractions, analysis of either fraction, after extensive dialysis revealed that neither fraction possessed the ability to induce increased rosette inhibition titres. In the study reported here, however, the supernatant and pellet fractions were assayed before dialysis, and although the pellet fraction did not induce increased inhibition titres, the supernatant fraction was as potent as the original pregnancy serum in expressing this activity (Fig. 1). As the extensively dialysed supernatant fraction did not display the ability to induce increased titres, active moieties of low molecular mass may have been lost from this fraction during the extensive dialysis procedure. The following experiment (Expt I) was undertaken to test this possibility. Samples of serum from day- 2 and day-10 pregnant mice were fractionated with $40 \%$ ammonium sulfate to yield supernatant and pellet fractions. The two fractions were dialysed in separate beakers against a 100-fold excess of PBS using nominal $8 \mathrm{kDa}$ cut-off dialysis membranes. At the completion of this first dialysis, aliquots of the retentates and the dialysates were collected and stored for later analysis in the rosette inhibition assay. The retentates were then subjected to a second and then a third dialysis, each time against a 100-fold excess of PBS and at the end of each dialysis step samples of the retentates and the dialysates were stored for analysis in the rosette inhibition assay.

Dialysis of the active supernatant fractions against 100-fold excess of PBS yielded retentate fractions (Fig. 1g) that were devoid of the capacity to induce increased inhibition titres. However, the dialysates (Fig. Id) collected from these first dialysis steps were extremely potent in inducing increased titres. Indeed, when it was taken into account that a 100-fold dilution was involved in generating the dialysates, these fractions were even more potent than the supernatant fractions from which they were derived. The derivation of these active, dialysate fractions from the supernatant fraction by the dialysis procedure suggests that low $M_{\mathrm{r}}$ active moieties (less than $8000 \mathrm{kDa})$ diffused from the supernatant fraction within the dialysis bag during the procedure.

However, such small molecules would be expected to equilibrate across the dialysis membrane during this procedure, and would therefore be present in equivalent concentrations in the dialysate and retentate fractions. Consequently the failure of the first retentate fractions to induce increased inhibition titres implies the presence in these fractions of substances ( $>8 \mathrm{kDa}$ ) that counteract the action of the low molecular mass active moieties (see further below). Active moieties were shown to be still present in these initial retentates when these retentates were dialysed again against a second 100-fold excess of PBS. After this second dialysis, the retentates were still devoid of activity (Fig. Ih), but the second dialysates (Fig. Ie) could induce increased inhibition titres with dose-response data consistent with that expected of a free equilibration of the low molecular mass active moieties during the second dialysis step. When the retentates were subjected to a third dialysis step, the dialysates obtained (Fig. If) were just active in the rosette inhibition assay, while the extensively $\left(10^{6}\right)$ dialysed supernatant retentates (Fig. 1i) were still devoid of this capacity. Comparison of the dose-response data for the three dialysate fractions (Figs $I d-f$ ) clearly indicates that low $M_{\mathrm{r}}$ active moieties are washed out during successive dialysis steps. During the three successive dialysis steps performed on the pellet fraction, no fractions were obtained that possessed the capacity to induce increased inhibition titres (Figs $1 \mathrm{j}-\mathrm{O}$ ). These 


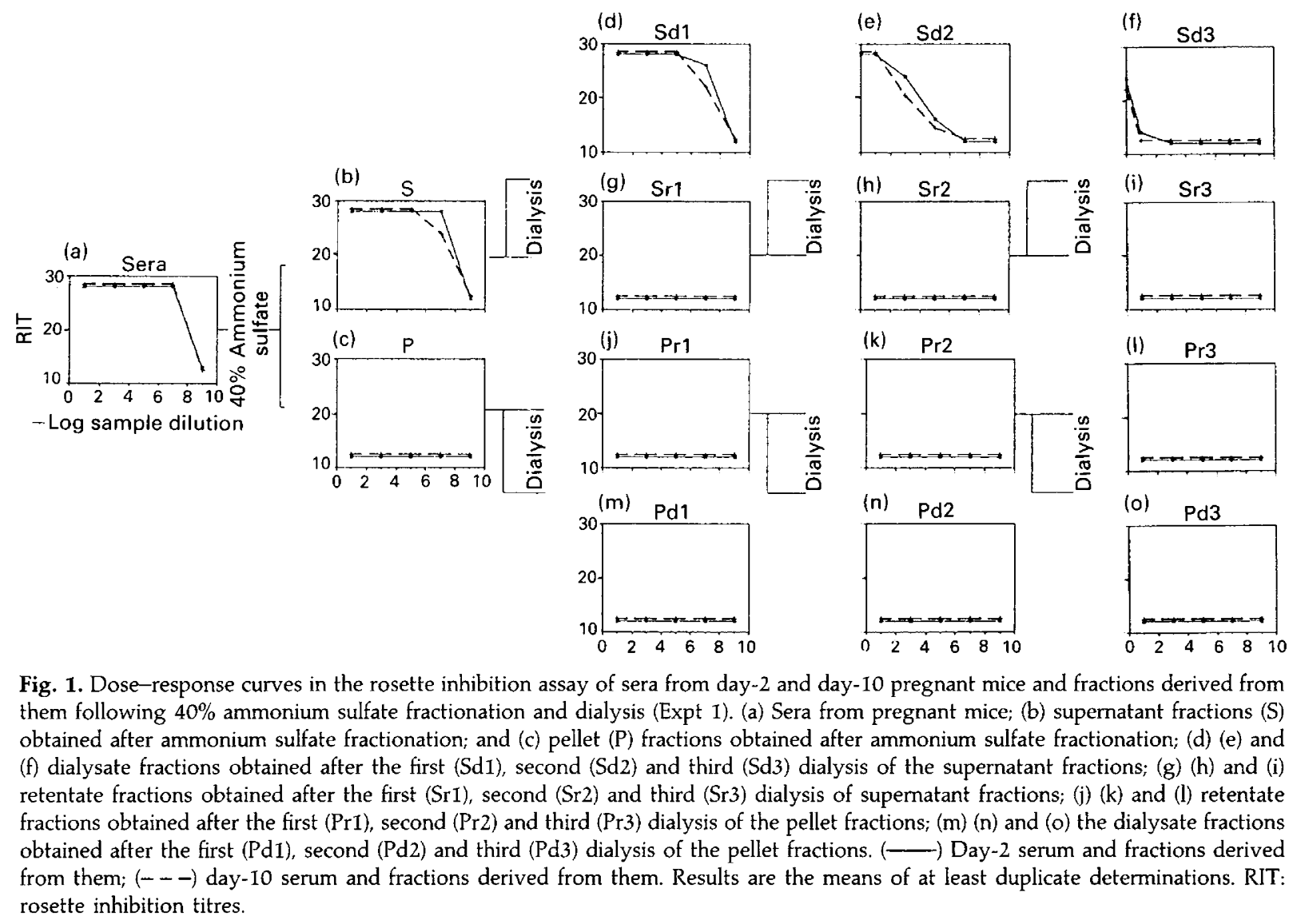

results indicate that low molecular mass active moieties are present in pregnancy sera and that they partition into the supernatant fraction following treatment with $40 \%$ ammonium sulfate. The approximate sizes of these low molecular mass active moieties were further assessed by dialysing supernatant fractions derived from day-10 pregnancy mouse sera using both $8 \mathrm{kDa}$ and $1 \mathrm{kDa}$ cut-off membranes against a 100-fold excess of PBS. Identical results were obtained using both dialysis membranes and the dialysate fractions were equally potent in inducing increased rosette inhibition titres, whereas the retentates were devoid of this capacity; a result indicating that the active moieties were less than $1 \mathrm{kDa}$ (data not shown).

As noted above, such small moieties would be expected to equilibrate across the membranes and be present in equivalent concentrations in both the dialysate and retentate fractions. However, while the initial dialysate fractions (Fig. 1d) were potent in inducing increased inhibition titres, the initial retentates (Fig. Ig) did not display this capacity. This finding suggests that the supernatant retentates contain substances $(>8 \mathrm{kDa}$ ) retained within the dialysis membranes that can counteract the action of the low molecular mass active moieties in a dose-dependent manner. Compared with the original supernatant fractions before dialysis, the concentration of low molecular mass moieties in the first retentates would have decreased 100-fold, whereas the concentration of potential counteracting substances (that are too large to equilibrate through the dialysis membrane) would be essentially unchanged. Consequently the ratio of counteracting substances to active moieties in the dialysed retentate fractions would be expected to be 100-fold greater than that in the undialysed supernatant fractions, which may account for the inability of the dialysed retentates to induce increased inhibition titres, even though they contain potentially active moieties. Samples of 1 in 10 dilutions of the first dialysates (Sd1, Fig. Id) (which contain active low molecular mass moieties) were mixed with samples of tenfold serial dilutions of the extensively dialysed supernatant retentate fractions ( $\mathrm{Sr} 3$, Fig. Ii) and the mixtures used in the rosette inhibition assay to test for the presence of counteracting substances in the supernatant retentate fraction. The extensively dialysed supernatant retentate fractions counteracted the capacity of the low molecular mass moieties in the initial $\mathrm{Sd} I$ dialysate fractions to induce increased inhibition titres in a dose-dependent manner (Fig. 2). At high ratios of retentate to dialysate, no increased inhibition titres were observed, but as the proportion of retentate to dialysate decreased, increased rosette inhibition titres were observed. Expression of activity by these mixtures was first observed when the ratio of retentate to low molecular mass moieties approached one, that is, when the proportions approximated to those that would be found in the original undialysed supernatant fractions.

Implicit in the above considerations has been the assumption that ammonium sulfate treatment was responsible for the appearance of the low molecular mass active moieties in the dialysates. This was tested directly by subjecting samples of untreated sera from mice at days 2, 7 and 10 of pregnancy to 


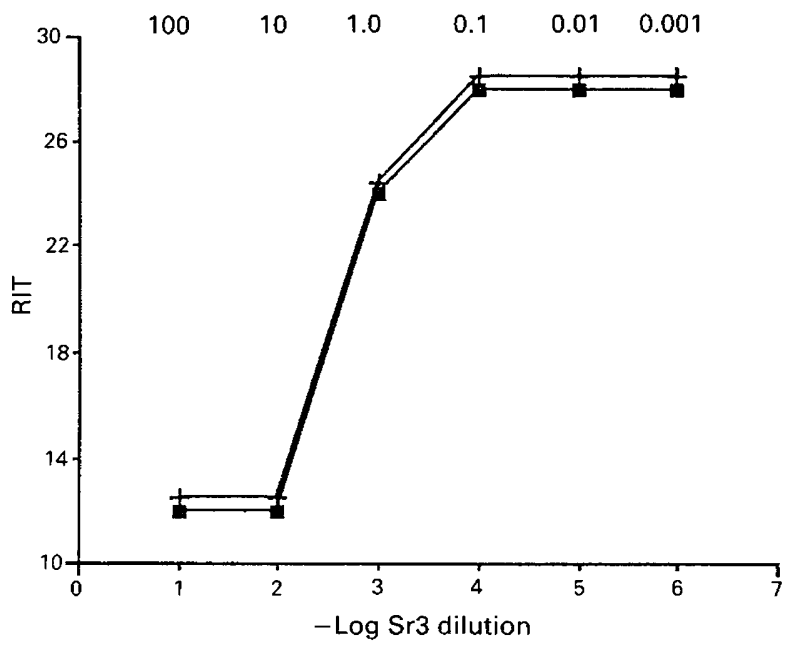

Fig. 2. Dose-response effects of the extensively dialysed supernatant retentate fractions ( $\mathrm{Sr} 3$ ) in counteracting the activity of the initial supernatant dialysate fractions $(\mathrm{Sd} 1)$ in the rosette inhibition assay. Two microlitres of 1 in 10 dilutions of the Sd1 fractions were mixed with $2 \mu \mathrm{l}$ of 1 in 10 serial dilutions of the Sr3 fractions, and the mixtures were applied to mouse spleen cells and the rosette inhibition titres determined. Numbers along the top of the figure indicate the ratio of $\mathrm{Sr} 3: \mathrm{Sd} 1$ components in the mixtures relative to the proportions in which these components occur in the original supernatant samples before dialysis. (E) Samples derived from sera from day-2 pregnant mice; $(+)$ samples derived from sera from day-10 pregnant mice. Results are the means of triplicate determinations. RIT: rosette inhibition titres.

the three-step dialysis procedure used above and samples of the dialysates and retentates were collected at each step and analysed in the rosette inhibition assay. The results obtained with the day-10 pregnancy serum sample were essentially identical to those obtained with samples from days 2 and 7 (Fig. 3). The first dialysis resulted in a slight loss of potency of the dialysed serum compared with the undialysed serum. The dialysate derived from this first dialysis step did display some capacity to induce increased inhibition titres (Fig. 3b), albeit with a potency many orders of magnitude less than that observed for dialysates derived from $40 \%$ ammonium sulfate supernatants of such pregnancy sera (compare Figs $1 b$ and $3 b$ ). The serum retentates obtained after the second and third dialysis steps showed no further loss of potency and were equivalent to the first serum retentate in their capacity to induce increased inhibition titres (Fig. 3a). The second and third dialysates (Fig. 3b) were devoid of the capacity to induce increased inhibition titres, indicating that active moieties were not washed out any further. Samples of the serum retentates after the third dialysis step were treated with $40 \%$ ammonium sulfate to yield supernatant and pellet fractions to test whether potentially active low molecular mass moieties were still present in the extensively dialysed serum samples. When analysed in the rosette inhibition assay before dialysis, the supernatant fraction was potent in inducing increased inhibition titres but the pellet fraction was devoid of this capacity (Fig. $3 c$ ). When the supernatant fraction was dialysed against a 100-fold excess of PBS, a dialysate fraction was obtained that was extremely potent in inducing increased inhibition titres
(Fig. 3d), indicating that potentially low molecular mass active moieties were present in the extensively dialysed serum samples presumably in tight association with molecules of greater molecular mass. Treatment with ammonium sulfate released the low molecular mass moieties from these associations and they partitioned into the supernatant fraction, where on subsequent dialysis, they were free to equilibrate across the dialysis membrane and so appear in the dialysates. This freedom to equilibrate across the dialysis membrane during the successive dialysis steps (see Figs $1 \mathrm{~d}-f$ ) during which the ammonium sulfate concentration in the supernatant retentate fractions was being lowered suggests that the larger molecular mass molecules with which the low molecular mass active moieties associate in serum were not in the supernatant fraction, but had partitioned into the pellet fraction during the ammonium sulfate fractionation.

\section{Cooperation between extensively dialysed pellet and supernatant retentate fractions derived from pregnancy sera in mimicking the action of sera from pregnant animals}

The observations of Clarke et al. (1980) were further tested and extended by combining the extensively dialysed pellet and supernatant retentate fractions from Expt I (Fig. 1), and the mixtures were tested for their capacity to induce increased inhibition titres. Although the individual retentate fractions alone were devoid of this capacity (see Figs $1 \mathrm{i}, \mathrm{l}$ ), the combinations of these extensively dialysed supernatant and pellet retentate fractions were effective in inducing increased inhibition titres. The dose-responses of the mixtures made by mixing equal amounts of each fraction and then applying serial dilutions of the mixtures to spleen cells before testing in the rosette inhibition assay are shown (Fig. 4a). Clearly, the combined pellet and supernatant retentate fractions were extremely potent in inducing increased inhibition titres. The dose-responses of the pellet retentate fractions in cooperating with a fixed amount of supernatant retentate fraction are shown (Fig. 4b) with the dose-responses of the supernatant retentate fractions in cooperating with a fixed amount of the pellet retentate fraction (Fig. 4c). These results clearly demonstrate that each fraction is extremely effective in cooperating with the other in allowing for the expression of increased inhibition titres.

The mode of action of these extensively dialysed retentate fractions in cooperating to allow for increased inhibition titres was investigated by testing the effects of the sequential order of addition of these fractions to spleen cells. When spleen cells were first incubated with a fixed amount of the supernatant retentate fractions, washed, and then incubated with serial dilutions of the pellet retentate fractions, and then again washed and tested in the rosette inhibition assay, increased inhibition titres were observed (Fig. 4d) with dose-responses similar to those obtained when mixtures of these components were added simultaneously to the spleen cells (compare with Fig. $4 \mathrm{~b}$ ). If the two-step protocol was performed in the reverse order (that is, a fixed amount of the pellet retentate fractions first, followed by serial dilutions of the supernatant retentate fractions) then no increases in inhibition titres above control values were seen (Fig. 4d). 

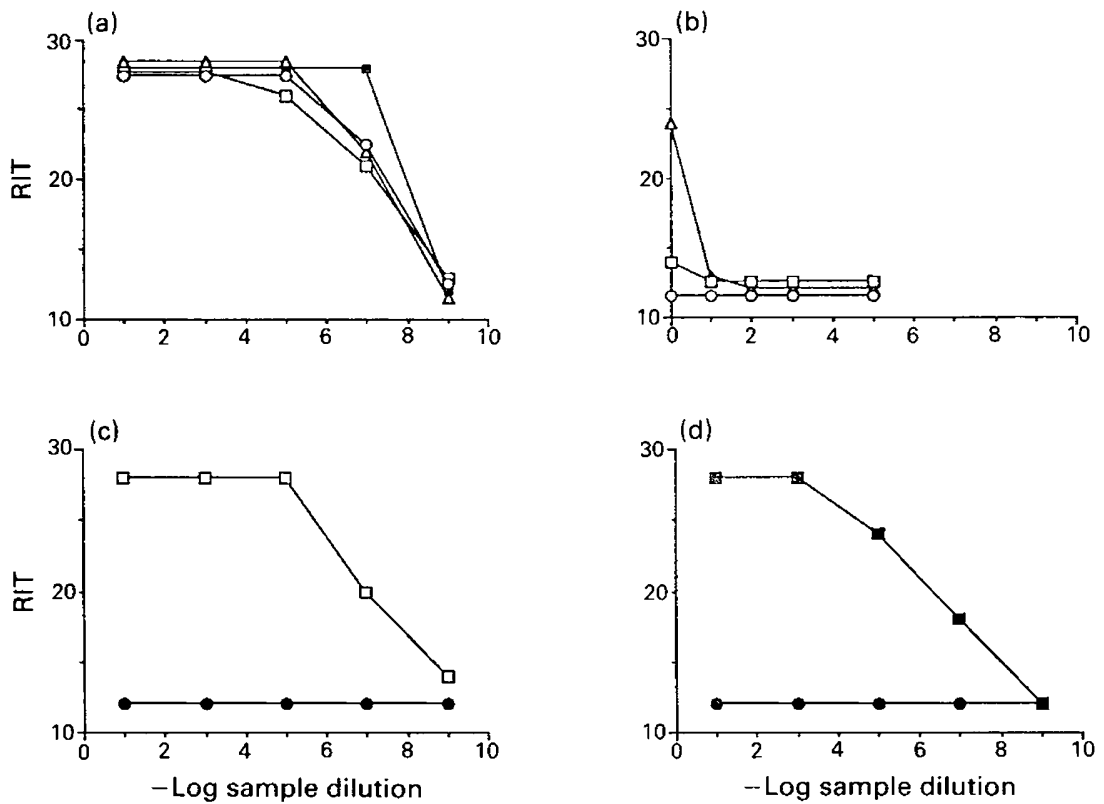

Fig. 3. Dose-response effects in the rosette inhibition assay of serum from day-10 pregnant mice and fractions derived from them by extensive dialysis followed by subsequent ammonium sulfate fractionation. (a) Serum from day 10 pregnant mice ( $\boldsymbol{\square}$ ) and retentates obtained after the first $(\triangle)$, second $(\square)$ and third $(O)$ dialysis steps; (b) dialysate fractions obtained after the first $(\triangle)$, second $(\square)$ and third $(O)$ dialysis of the serum sample; (c) supernatant $(\square)$ and pellet $(\bigcirc)$ fractions obtained after ammonium sulfate fractionation of the extensively dialysed serum sample and (d) dialysate $(\boldsymbol{\square})$ and retenate $(\boldsymbol{O})$ fractions obtained on dialysis of supernatant fraction against 100-fold excess of PBS. Results are means of duplicate determinations. RIT: rosette inhibition titres.

On the basis of experimental data provided in this report, the following general conclusions can be made. (i) Pregnancy sera contain low molecular mass moieties potentially active in the rosette inhibition assay, some of which can be removed by dialysis of the sera. However, most, or at least the most potent, of these moieties in pregnancy sera are associated with macromolecular components and are not removed by simple dialysis. (ii) Treatment of sera from pregnant mice with $40 \%$ ammonium sulfate liberates the bound, low molecular mass moieties from their associations with macromolecular components and they partition into the supernatant fraction following centrifugation, whereas the macromolecular components, to which they were bound, partition into the pellet. (iii) When the supernatant fractions are dialysed, the low molecular mass moieties freely equilibrate with the dialysate and can be filtered out of the supernatant fraction by successive dialyses. (iv) Macromolecular components in the supernatant fraction can counteract the action of the low molecular mass moieties in the rosette inhibition assay in a dose-dependent manner; and (v) the macromolecular components of the supernatant and pellet fractions freed of any association with molecular mass moieties cannot alone induce increased inhibition titres; however, when combined, components in the pellet fraction cooperate with components in the supernatant fraction resulting in increased inhibition titres. Further studies on the mode of action of these macromolecular components in the pellet and supernatant retentate fractions are described by Orozco $e t$ al. (1994).
Ability of the low molecular mass moieties to associate with components in the pellet retentate fraction

Before examining the modes of action of these extensively dialysed pellet and supernatant retentate fractions derived from pregnancy sera, it is important to first address some apparent conflicts between the results reported here and the interpretations and some of the observations and interpretations recently reported by Cavanagh et al. (1991). Cavanagh et al. (1991) reported that extensively dialysed supernatant retentate fractions from sera of day 2 pregnant animals could not induce increased inhibition titres. However, in contrast to our results, they reported that the extensively dialysed pellet retentate fraction derived from day- 2 sera possessed the capacity to induce increased inhibition titres, albeit with a characteristic bell-shaped dose-response curve, where very low and very high doses failed to induce increased inhibition titres, but intermediate doses did. Furthermore, they reported that the extensively dialysed pellet and supernatant retentate fractions derived from sera of day-10 pregnant mice could increase titres, with both displaying bell-shaped dose-response curves. These, and other results, such as the shape of the dose-response profiles of the recombined pellet and supernatant retentate fractions are in apparent contrast to the results reported here for apparently comparable fractions. In the experiments reported here, the extensively dialysed pellet and supernatant retentate fractions derived from sera of day- 2 and day-10 pregnant mice could not induce increased inhibition titres over 

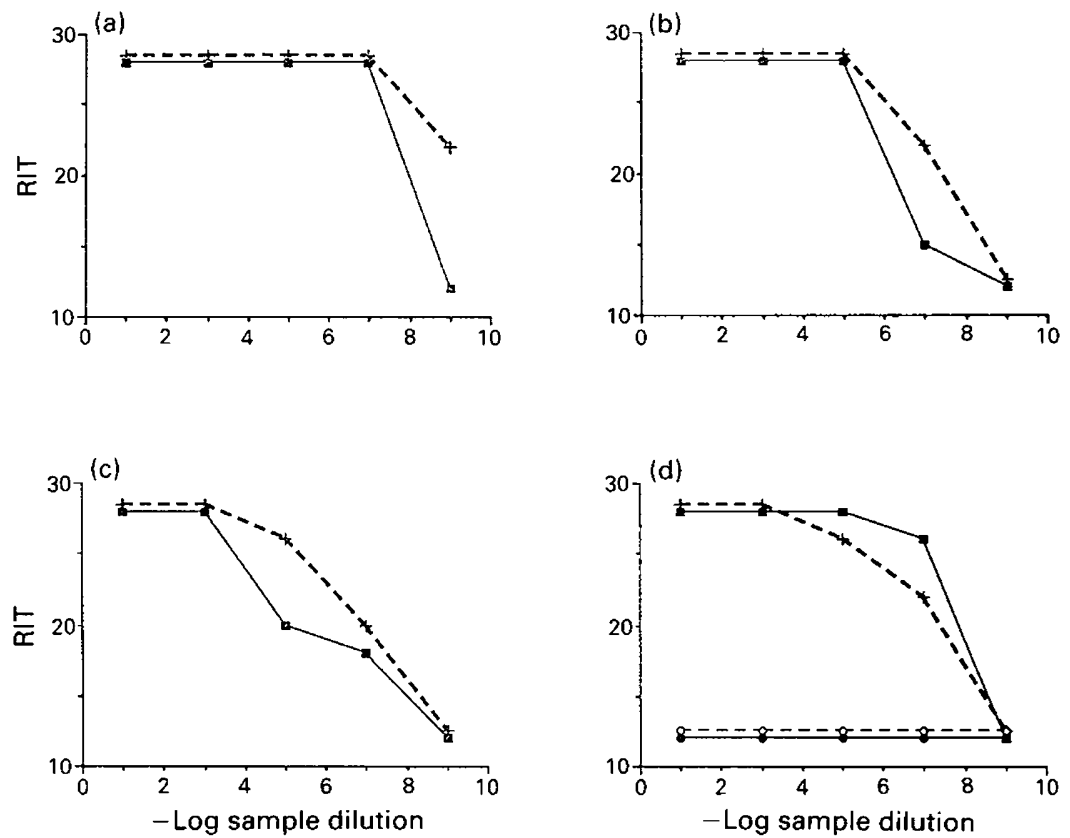

Fig. 4. Dose-response curves in the rosette inhibition assay of mixtures made by combining the extensively dialysed supernatant $(\mathrm{Sr} 3)$ and pellet $(\operatorname{Pr} 3)$ retentate fractions derived from mouse pregnancy sera. (a) Dose-response curves of mixtures made by combining equal amounts of pellet and supernatant retentate fractions derived from serum from ( $\boldsymbol{\square}$ ) day-2 and $(+)$ day-10 pregnant mice; (b) effect of different doses $(2 \mu \mathrm{I}$ of indicated dilutions) of pellet retentates in cooperating with a fixed amount ( $2 \mu \mathrm{l}$ of 1 in 10 dilutions) of supernatant retentate fractions, ( $\boldsymbol{\square}$ ) day- 2 and $(+)$ day-10 derived samples; (c) effect of different doses ( $2 \mu$ l of indicated dilutions) of supernatant retentates in cooperating with a fixed amount ( $2 \mu \mathrm{l}$ of 1 in 10 dilutions) of pellet retentates fractions, $(\boldsymbol{\square})$ day-2 and $(+)$ day-10 derived samples; (d) dose-response effects of pellet retentate fractions in cooperating with supernatant retentate fractions in a two-step incubation protocol in which cells were incubated with $2 \mu \mathrm{l} I$ in 10 dilutions of supernatant retentate fractions, washed, then incubated with $2 \mu \mathrm{l}$ of indicated dilutions of the pellet retentate fractions before testing in the assay, ( $\boldsymbol{a}$ ) day- 2 and $(+)$ day-10 derived samples; dose-response effects of supernatant retentate fractions in cooperating with pellet retentate fractions in a two step incubation protocol in which cells were incubated with $2 \mu \mathrm{l}$ of 1 in 10 dilutions of pellet retentate fractions, washed, then incubated with $2 \mu \mathrm{l}$ of indicated dilutions of the supernatant retentate fractions before testing in assay, (O) day-2 and $(O)$ day-10 derived samples. RIT: rosette inhibition titres.

an extensive dose range, comparable to that used by Cavanagh et al. (1991). When combined, these retentate fractions induced increased inhibition titres, but with simple, open dose-response curves.

We looked for possible protocol differences either in the assay procedures or in the preparation of the samples to explain these differences. Compared with the assay procedure used by Cavanagh et al. (1991) we used (i) a different lysis procedure in the preparation of the spleen cells; (ii) human serum as a source of complement not guinea-pig serum and (iii) the cell incubation step was performed in PBS not HBSS. Although these modifications of the assay have been documented (Orozco et al., 1986; Clarke et al., 1987) as valid and contributing to the reliability of the assay in the detection of 'early pregnancy factor' activity, we found that the assay procedure was not a possible cause of the difference between our observations and those of Cavanagh $e$ t al. by reassaying our samples using the assay protocol of Cavanagh et al. (1991).
The results were the same as those obtained by our usual assay procedure. Consequently, any protocol differences of significance must have been in the procedures used to prepare the fractions Cavanagh and co-workers did not define the extent, or provide many details of the dialysis procedure, nor did they assay the supernatant or pellet fractions before dialysis and they did not assay the dialysates. Consequently they failed to detect the presence of low molecular mass active moieties in the sera and the supernatant fractions derived from them. This failure to appreciate the potential presence of low molecular mass moieties of consequence to the phenomena under study may have led these investigators to dialyse the pellet and supernatant fractions from the same, or even different, sera against the same solution in the same container. If this was the case, this procedure is different from our protocol in which each fraction was dialysed in a separate beaker and would have significant consequence. If the pellet and supernatant fractions were dialysed against the same solution in the same container, 




Fig. 5. Dose-response curves in the rosette inhibition assay of fractions derived from sera of day-2 and day-10 pregnant mice following $40 \%$ ammonium sulfate fractionation and dialysis in which the pellet and supernatant fractions from a given serum were dialysed against the same PBS solution in the same beaker. (a) Pregnancy sera; (b) supernatant (S) fractions and (c) pellet (P) fractions obtained after ammonium sulfate fractionation; (d) (e) and ( $\mathrm{f}$ ) dialysate fractions obtained after the first $\left(\mathrm{d} 1^{\prime}\right)$, second $\left(\mathrm{d} 2^{\prime}\right)$ and third $\left(\mathrm{d} 3^{\prime}\right)$ dialysis steps; (g) (h) and (i) supernatant retentate fractions obtained after the first $\left(\mathrm{Sr} \mathrm{I}^{\prime}\right)$, second $\left(\mathrm{Sr} 2^{\prime}\right)$ and third $\left(\mathrm{Sr} 3^{\prime}\right)$ dialysis steps; (j) (k) and (l) pellet retentate fractions obtained after the first $\left(\operatorname{Pr} 1^{\prime}\right)$, second $\left(\operatorname{Pr} 2^{\prime}\right)$ and third $\left(\operatorname{Pr} 3^{\prime}\right)$ dialysis steps; $(m)$ mixtures formed by combining equal amounts of the extensively dialysed pellet $\left(\operatorname{Pr} 3^{\prime}\right)$ and supernatant $\left(\operatorname{Sr} 3^{\prime}\right)$ retentate fractions. $(\longrightarrow$ ) Day- 2 serum and fractions derived therefrom; (- - ) day-10 serum and fractions derived therefrom. Results are the means of at least duplicate determinations. RIT: rosette inhibition titres.

the low molecular mass active moieties, which we have shown to be present, largely in a bound form in serum, but which are liberated into the supernatant fraction by ammonium sulfate treatment, would be free to equilibrate with both retentate fractions, possibly reassociating with macromolecules in the pellet fractions during the dialysis procedure. Clearly, this could change the nature of the extensively dialysed fractions. The pellet and supernatant fractions were dialysed against the same solution in the same beaker, to investigate this possibility.

In Expt 2, pellet and supernatant fractions were prepared from sera of day 2 and day 10 pregnant mice and then subjected to a dialysis protocol (using nominal $1 \mathrm{kDa}$ cut-off membranes) similar to that used in Expt 1 ; the only difference was that after the pellet and supernatant fractions derived from each sera were placed in separate dialysis bags, the pellet and supernatant fractions from a given serum were dialysed together in the same beaker against the same PBS solution. As for Expt 1, three successive dialysis steps were then carried out and samples of the retentates and dialysates were kept for analysis after each step. The dose-response curves of these samples from Expt 2 in the rosette inhibition assay are shown (Fig. 5). As noted previously, the supernatant fractions before dialysis were extremely potent in inducing increased inhibition titres, whereas the pellet fractions did not show this capacity (Figs $5 \mathrm{~b}, \mathrm{c}$ ). Dialysis of these supernatant and pellet fractions against the same 100-fold excess of PBS solution in the same beaker yielded supernatant retentate fractions, pellet retentate fractions and dialysate fractions. As in the separate beaker experiments (Fig. 1) the initial supernatant retentate fractions (Fig. $5 \mathrm{~g}$ ) derived from day- 2 and day-10 sera could not induce increased inhibition titres. However, in contrast to the results obtained in the separate beaker dialysis experiment, the initial pellet retentate fractions (Fig. $5 \mathrm{j}$ ) from the present experiment could induce increased inhibition titres, and the dose-response curves showed the characteristic bell shapes noted by Cavanagh et al. (1991) for their extensively dialysed pellet fractions. The subsequent supernatant and pellet retentate fractions obtained after the second and third dialysis steps maintained the same characteristics as noted for the respective initial retentate fractions: namely the successive supernatant retentate fractions (of day- 2 and day-10 sera) could not induce increased inhibition titres over an extensive range of dilutions (Figs $5 \mathrm{~h}, \mathrm{i}$ ), whereas the successive pellet retentate fractions could induce increased inhibition titres over a limited, intermediate range of dilutions giving characteristic bell-shaped dose-response profiles, which did not change much with successive dialysis steps (Fig. $5 \mathrm{k}, \mathrm{l}$ ). The dialysate fractions obtained after the initial dialysis step could induce increased inhibition titres (Fig. 5d) indicating the presence of low molecular mass active moieties. However, compared with the dialysate fractions obtained on dialysing the supernatant fractions alone in separate beakers, the initial dialysates obtained when the pellet and supernatant fractions were dialysed in the same beaker were considerably less potent (compare Figs 5d and $\mathrm{Id}$ ). In addition, the dialysate fractions obtained after the second and third dialysis steps were devoid of activity 




Fig. 6. Dose-response curves in the rosette inhibition assay of the extensively dialysed pellet retentate fractions and fractions derived from them following ammonium sulfate fractionation and subsequent dialysis. (a) Extensively dialysed pellet retentate fractions; (b) the supernatant and (c) pellet fractions obtained after treatment with $40 \%$ ammonium sulfate; (d) the retentate and (e) the dialysate fractions obtained after dialysis of the supernatant fraction against 100-fold excess volume of PBS; $(\mathrm{f})$ the retentate and $(\mathrm{g})$ dialysate fractions obtained after dialysis of the pellet fraction and $(\mathrm{h})$ the mixture formed by combining equal amounts of the dialysed pellet retentate (from $\mathrm{f}$ ) and the supernatant dialysate (from e) fractions. (-) Serum from day-2 pregnant mice and fractions derived therefrom; (- - ) day-10 serum and fractions derived therefrom. Results are the means of at least duplicate determinations. RIT: rosette inhibition titres.

(Figs $5 \mathrm{e}, \mathrm{f}$ ). This is in contrast to the apparent washing out of active moieties from the supernatant fractions when dialysed alone in a separate beaker in dialysis Expt 1 . When the extensively dialysed pellet retentate (Fig. 5I) and supernatant retentate (Fig. 5i) fractions obtained from dialysis Expt 2 were combined, the mixtures were effective in inducing increased inhibition titres (Fig. $5 \mathrm{~m}$ ), and open dose-response curves were observed in contrast to the bell-shaped response curve seen with the dialysed pellet retentate fractions alone. The potencies of these mixtures (particularly those from the day- 2 derived samples) were somewhat lower than those of the mixtures formed by combining the separately dialysed pellet and supernatant retentate fractions from Expt 1.

The relatively low potency of the initial dialysate fractions, and the subsequent absence cf any activity in the second and third dialysate fractions from dialysis Expt 2, suggested that the low molecular mass active moieties, initially present in a free form in the supernatant fractions, had reassociated with macromolecular components in either the supernatant or pellet retentate fractions, during the combined dialysis procedure used in this experiment. As the results of Expt 1 revealed no evidence of an association between the low molecular mass active moieties and the macromolecular components of the supernatant retentate fractions, it is most likely that these low molecular mass active moieties had become reassociated with macromolecular components in the pellet retentate fractions, as the ammonium sulfate concentration in those fractions is lowered during the dialysis procedure. Such a reassociation could account for the activity expression of the extensively dialysed pellet retentate fractions, and their characteristic 
dose-response profiles. Indeed these bell-shaped doseresponse curves were similar to the activity characteristics of the preparations previously isolated from ovine (Clarke et al., 1987) and human (Di Trapani $e t$ al., 1991) placental extracts, in which it has been shown that activity is due to the isolation of low molecular mass active moieties in association with a protein identified as thioredoxin.

If such a reassociation had occurred during the preparation of the extensively dialysed pellet retentate fractions in dialysis Expt 2, treatment of these pellet retentate fractions with $40 \%$ ammonium sulfate should release any low molecular mass active moieties back into the supernatants as the protein components are again precipitated. This proposition was tested by adding ammonium sulfate to $40 \%$ of samples of the extensively dialysed pellet retentate fractions from dialysis Expt 2, causing precipitation of $95-99 \%$ of the protein. After centrifugation, the supernatant fractions were collected and the pellet fractions dissolved in a volume of PBS equivalent to the volume of the original sample. While the original samples displayed the capacity to induce increased inhibition titres with characteristic bell-shaped dose-response curves (Fig. 6a), the pellet fractions obtained after ammonium sulfate treatment (which contain nearly all the protein originally present in the samples) could not induce increased titres when tested over comparable dose ranges (Fig. 6c). The supernatants, however, were potent in inducing increased inhibition titres and did so with open dose-responses (Fig. 6b). These pellet and supernatant fractions were then dialysed (in nominal $\mathrm{I} \mathrm{kDa}$ cut-off membranes) against a 100-fold excess of PBS in separate beakers, yielding retentates and dialysates that were then tested in the rosette inhibition assay. Neither the retentates nor dialysates derived from the pellet fractions had any activity in the assay (Fig. 6f, g). However, the dialysis of the originally active supernatant fractions yielded retentate and dialysate fractions that were equipotent in inducing increased inhibition titres (Fig. 6d, e), demonstrating the presence in the original supernatant fractions of low molecular mass active moieties, which freely equilibrated throughout the retentate and dialysate compartments during the dialysis procedure. The 100-fold decrease in the limiting positive dose of these fractions compared with that of the original supernatant samples (compare Fig. 6d, e with Fig. 6b) was consistent with this conclusion. The dialysate fractions derived from the supernatants had open dose-response curves when tested alone (Fig. $6 e)$, but when reconstituted with an equal volume of the dialysed pellet retentate fraction (Fig. 6f), the dose-response characteristics changed and the mixtures displayed (Fig. 6h) the bell-shaped dose-response profiles characteristic of the original unfractionated pellet fractions (compare Fig. 6h with e, a) indicating that the association of the low molecular mass moieties with the macromolecular components of the pellet fraction modified their dose-response behaviour. An identical experiment carried out with extensively dialysed pellet fractions from Expt I showed no evidence of low molecular mass moieties in those preparations.

Comparison of the results of Expt 1 with those of Expt 2 clearly reveal that the procedures used in dialysing the pellet and supernatant fractions derived from ammonium sulfate fractionation of sera of pregnant mice can influence the properties of the resulting extensively dialysed retentate fractions. Although Expt 2 did not reproduce all the observations of Cavanagh et al. (1991), it did reproduce a key finding, on which much of their interpretation was based, namely an extensively dialysed pellet retentate fraction active in the rosette inhibition assay with characteristic bell-shaped doseresponse curves. While Cavanagh et al. (1991) interpreted similar observations as indicating the partitioning of some pregnancy-specific protein into the pellet fractions, the data we have provided indicate that this does not occur, at least not for the extensively dialysed pellet retentate fractions produced in Expt 2. For these it has been clearly demonstrated that expression of activity was due to the presence of low molecular mass moieties that reassociated with macromolecular components in the pellet retentate fractions when the pellet and supernatant fractions were dialysed together in the same beaker. Although specific details of the dialysis procedures used by Cavanagh et al. (1991) are unavailable, it is possible that differences in dialysis procedures could account for the apparent conflict between their results and those we obtained in Expt 1.

This work was supported in part by the National Health and Medical Research Council of Australia.

\section{References}

Bessho T, Taira S, Ikuma K, Shigeta M, Koyama K and Isojima S (1984) Detection of early pregnancy factor in the sera of conceived women before implantation Acta Obstetrica Gynaecology Japan 36 391-396

Bose R, Chen H, Sabaddidni E, McCoshen J, Mahadevan M and Fleetham J (1989) Purified human early pregnancy factor from pre-implantation embryo possesses immunosuppressive properties American Journal of Obstetrics and Gynecology $160954-960$

Cavanagh AC (1984) Production in vitro of mouse early pregnancy factor and purification to homogeneity Journal of Reproduction and Fertility 71 581-592

Cavanagh AC (1985) Purification and partial characterisation of EPF. In Early Pregnancy Factors pp 179-189 Eds F Ellendorf and E Koch. Perinatology Press, New York

Cavanagh AC, Morton H, Athanassas-Platsi K, Quinn K and Rolfe BE (1991) Identification of a putative inhibitor of early pregnancy factor in mice joumal of Reproduction and Ferility 91 239-248

Chard T and Grudzinkas JG (1987) Early pregnancy factor Biological Research Progress 8 53-56

Clarke FM (1992) Identification of molecules and mechanisms involved in the 'early pregnancy factor' system Reproduction, Fertility and Development 4 $423-433$

Clarke FM and Wilson S (1985) In search of EPF. In Early Pregnancy Factors pp 165-177 Eds F Ellendorf and E Koch. Perinatology Press, New York

Clarke FM, Morton $\mathrm{H}$ and Clunie GJA (1978) Detection and separation of two serum factors responsible for depression of lymphocyte activity in pregnancy Clinical Experimental Immunology 32 318-323

Clarke FM, Morton H, Rolfe BE and Clunie GJA (1980) Partial characterisation of early pregnancy factor in the sheep Joumal of Reproductive Immunology $\mathbf{2}$ $151-162$

Clarke FM, Wilson S, McCarthy R, Perkins A and Orozco C (1987) Large scale isolation of RIT-active polypeptides from ovine placental extracts Journal of Reproductive Immunology 10 133-156

Clarke FM, Orozco C, Perkins AV and Cock I (1990a) Partial characterisation of the PAF-induced soluble factors which mimic the activity of 'early pregnancy factor' Journal of Reproduction and Fertility $\mathbf{8 8} 459-466$

Clarke FM, Cock I, Perkins A and Orozco C (1990b) Leukotrienes are active in the rosette inhibition assay mimicking the action of 'early pregnancy factor' Biochemical and Biophysical Research Communications $167535-542$

Clarke FM, Orozco C, Perkins AV, Cock I, Tonissen KF, Robins AJ and Wells JRE (1991) Identification of molecules involved in the 'early pregnancy factor' phenomenon Joumal of Reproduction and Fertility 93 525-539 
DiTrapani G, Orozco C, Perkins A and Clarke F (1991) Isolation from human placental extracts of a preparation possessing 'early pregnancy factor' activity and identification of the polypeptide components Human Reproduction 6 450-457

Klima F, Tiemann U, Schadow D, Fasinski M, Savoly SB, Loose R and Pitra C (1992) Bovine early pregnancy factor (EPF) activity dependent on a $67-\mathrm{kDa}$ polypeptide Joumal of Reproductive Immunology 21 57-90

Mehta AR, Eessalu TE and Aggarwal BB (1989) Purification and characterisation of early pregnancy factor from human pregnancy sera Journal of Biological Chemistry 264 2266-2271

Mesrogli M, Schneider J and Maas DHA (1988) Early pregnancy factor as a marker for the earliest stages of pregnancy in infertile women Human Reproduction 3 113-115

Morton H (1984) Early pregnancy factor (EPF): a link between fertilization and immunomodulation Australia Journal of Biological Sciences 37 393-407

Morton H, Hegh V and Clunie GJA (1974) Immunosuppression detected in pregnant mice by the rosette inhibition test Nature 249 459-460

Morton H, Hegh V and Clunie GJA (1976) Studies of the rosette inhibition test in pregnant mice: evidence of immunosuppression? Proceedings of the Royal Society London B 193 413-419

Morton H, Rolfe B, Clunie GJA, Anderson MJ and Morrison J (1977) An early pregnancy factor detected in human serum by the rosette inhibition test Lancet i 394-397

Morton H, Rolfe BE, McNeill L, Clarke P, Clarke FM and Clunie GJA (1980) Early pregnancy factor: tissues involved in its production in the mouse Journal of Reproductive Immunology 2 73-82

Morton H, Rolfe B and Cavanagh AC (1987) Ovum factor and early pregnancy factor Current Topics in Developmental Biology 23 73-92

Orozco C, Perkins T and Clarke FM (1986) Platelet-activating factor induces the expression of early pregnancy factor activity in female mice journal of Reproduction and Fertility 78 549-555
Orozco C, Cock I, Perkins AV and Clarke FM (1990) Platelet-activating factor and serum components from oestrous mice co-operate to mimic the activity of 'early pregnancy factor' in the rosette inhibition assay Journal of Reproduction and Fertility 88 447-457

Orozco C, Clark IQ, Cock IE and Clarke FM (1994) A description of the basic system of components in pregnant mice sera responsible for early pregnancy factor activity Joumal of Reproduction and Fertility $100291-297$

Rolfe BE (1982) Detection of fetal wastage Fertility and Sterility 37 655-660

Rolfe BE, Morton H and Clarke FM (1983) Early pregnancy factor is an immunosuppressive contaminant of commercial preparations of human chorionic gonadotrophin Clinical Experimental Immunology 51 45-52

Rolfe BE, Cavanagh A, Forde C, Bastin F, Chen C and Morton H (1984) Modified rosette inhibition test using mouse lymphocytes for detection of early pregnancy factor in human serum Journal of Immunological Methods 70 1-1I

Smart YC, Roberts TK, Clancy R and Cripps AW (1981) Early pregnancy factor its role in mammalian reproduction - research review Fertility and Sterility 35 $397-402$

Sueoka K, Kusama T, Baba J, Wallach EE and Iizuka R (1989a) Biochemical consideration of human early pregnancy factor (EPF). In Development of Preimplantation Embryos and their Environment pp 317-329 Eds K Yoshimaga and M Takahide. Alan R Liss Incorporated, New York

Sueoka K, Dharmarajan AM, Miyazaki T, Atlas SJ and Wallach EE (1989b) In vivo and in vitro determination of components of rabbit early pregnancy factors Journal of Reproduction and Fertility 87 47-53

Whyte A and Heap RB (1983) Early pregnancy factor Nature 304 121-122

Wilson S, McCarthy R and Clarke FM (1983) In search of early pregnancy factor: isolation of active polypeptides from pregnant ewes' sera Journal of Reproductive Immunology 5 275-286

Wilson S, McCarthy R and Clarke FM (1984) In search of early pregnancy factor: characterisation of active polypeptides isolated from pregnant ewes' sera Journal of Reproductive Immunology $6253-260$ 